\title{
Arbor
}

\section{La Real Armería de Madrid}

\author{
Alvaro Soler del Campo
}

Arbor CLXIX, 665 (Mayo 2001), 143-161 pp

La creación de la Real Armería, como Colección, tiene su origen en las disposiciones testamentarias de Felipe II, quien en su testamento de 1594 y en el codicilo de 1597, ordenó que la Armería no pudiera ser vendida después de su muerte, según era costumbre, para pagar las deudas terrenales y espirituales del difunto. El 6 de marzo de 1594 Felipe II ordenó en su testamento que al príncipe Felipe se de libremente un diamante Rico que yo auia dado a su madre y de todo lo demas que me pertenesçe y dexare fuera de lo del Armeria cauallos $y$ pinturas y otras cosas ordinarias que quedaren puestas en las casas que tambien le doy libremente ... paresciendo que seran buenas para el serviçio del Principe Don Phelipe mi hijo y de nuestros sucessores le sean dadas y las pueda tomar en su precio y valor moderado a arbitrio de mis testamentarios. Con ello vinculó la Armería al futuro Felipe III y a sus sucesores, evitando que la Colección pudiera ser vendida en almoneda pública. El 23 de agosto de 1597 confirmó su última voluntad en el codicilo de su testamento, pero en esta ocasión se especificó el contenido de la Armería como «todo lo que en ella se hallare de la misma manera que esta puesto en su sala en Madrid $y$ con los aderesços de cauallos tanto Jaezes dela gineta como guarniçiones dela brida cubiertas y lo demas que entrambas sillas tocare que esta en el guadarnes».

Estas disposiciones, y el haber sido respetadas por sus descendientes, implicaron que la Colección fuera considerada como inalienable de la Corona de España. La decisión de Felipe II de mantener unido todo este conjunto de armas se debe a dos motivos: en primer lugar la Armería tenía un gran valor simbólico y sentimental, tanto por ser una representación dinástica de la Casa de Austria, como por contener 
las armas de su padre, a quien admiraba; en segundo lugar, se trataba de una Colección excepcional de armas de lujo, de un gran valor material, que debía ser conservada apropiadamente. Sus sucesores fueron enriqueciéndola con parte de sus armerías personales y otras armas de diversa procedencia. Con todo, no se trata de una colección dinástica en sentido estricto, ya que en ella no se han preservado todas las armas pertenecientes a los Monarcas Españoles ni había una voluntad determinante de que así sucediera. La historia de la Colección está jalonada por entradas y salidas de objetos, resultado de las más diversas circunstancias, como las dos armaduras de Carlos V y Felipe II regaladas por este último al Archiduque Fernando del Tirol, para su famosa "Armería de los Héroes» del castillo de Ambras, en Innsbruck. El hecho de existir cierta continuidad en la representación, más o menos precisa de los diferentes reinados, le ha conferido cierto carácter dinástico derivado de su formación a lo largo del tiempo, no porque hubiera una clara voluntad o una norma que así lo indicara.

La decisión de otorgar un trato preferente a la Armería se remonta, al menos, al fallecimiento de Carlos V, acaecido el 21 de septiembre de 1558. A finales de 1559 ya se había hecho saber a los testamentarios del Emperador la decisión del nuevo Rey de tomar para sí la Armería. El precio final se fijó en 12.000 ducados, cifra claramente ventajosa para Felipe II. La armería del Emperador había llegado, en su mayor parte, desde Bruselas a España a través del puerto de Laredo, en septiembre de 1556, desde donde pasó a Valladolid. A su muerte sus armas se encontraban dispersas entre Valladolid, tanto en la nueva armería situada en las casas que fueron del Comendador Cobos como en el Convento de San Pablo, en la fortaleza de Simancas, $y$, en menor medida, en el Monasterio de Yuste, y, posiblemente, en el Alcázar de Madrid.

El establecimiento de la Corte en Madrid explica que, en el mes de julio de 1562, Felipe II ya hubiera decidido que se hiciera en Madrid vna muy buena armeria sobre las cauallerizas $q$ agora estan hechas para que se puedan traer a ella las armas que estan en uallid. y poner las demas que aca tiene Su magd, determinación que pudo haber sido, sin embargo anterior. La construcción del edificio que se conocería como «la Armería» fue decidida, al parecer, en 1553, para dotar al Alcázar de unas nuevas Caballerizas ${ }^{1}$. La concepción del mismo se debe, probablemente, al propio Felipe II a juzgar por un croquis de su puño conservado en el Archivo General de Simancas. El edificio fundacional era rectangular, de dos plantas, con un tejado a dos aguas abuhardillado y frontones escalonados en ambos testeros. La planta 
inferior, destinada a caballerizas, tenía tres naves, mientras que la planta superior, donde se instaló la Armería, era completamente diáfana. El interior de la sala se terminó en 1565. Estaba encalada y decorada con un zócalo de azulejo de Talavera procedente del alfar de Juan Florez. Las armas se custodiaban dentro de grandes «cajones» de madera, es decir, grandes armarios parecidos a guardarropías. La distribución de las armas en la sala fue pensada concienzudamente. Las armas de mayor categoría se guardaron dentro de los cajones. Las armas de fuego, la ballestería, y, en menor medida, algunas armas blancas y las de asta pequeñas, en lanceras sobre las ventanas. Las restantes armas de asta ocuparon los testeros de la sala y de los cajones. En el testero occidental destacaban dos pequeñas piezas de artillería y cuatro trineos con las guarniciones de sus tiros.

Los criterios de distribución y ordenación interior de los cajones fueron más complejos. El primer criterio de distribución atendía a los propietarios de las armas. Las de Carlos V ocuparon, fundamentalmente, los ocho primeros cajones situados en lateral meridional, mientras que las de Felipe II se guardaron frente a las de su padre en la pared septentrional. De acuerdo con el segundo criterio, determinados cajones albergaron el conjunto de armaduras, piezas de refuerzo, arreos y vestidos que constituían cada uno de los arneses de Carlos V y Felipe II. El tercer criterio, más amplio, respondió a los tipos de objetos, tanto desde un punto de vista formal como material. De esta manera se establecieron cajones que guardaban un sólo tipo de armas, dedicados por ejemplo a armas blancas, a cotas de malla, u otros objetos con rasgos comunes como era su técnica decorativa en el caso de las armas decoradas en ataujía. Otros cajones custodiaban armas de especial interés para la dinastía, como el dedicado a los trofeos de Mülhberg y Pavía, o el que agrupaba armas de personajes legendarios o de cierta importancia simbólica, como el estoque de ceremonia de los Reyes Católicos, el estoque enviado por Clemente VII a Carlos V, las armaduras enviadas a Felipe II por el shogun Toyotomi Hideyosi, o las espadas atribuídas al Cid, al Gran Capitán, a Roldán y a Boabdil.

El núcleo principal de la Colección actual se corresponde con la armería custodiada por Felipe II cuando estableció la Corte en Madrid, compuesta por su armería personal, pero sobre todo por la de su padre, el Emperador Carlos V, quien a su vez había conservado armas pertenecientes a su propio padre, el Rey Felipe I de Castilla y a sus abuelos, Fernando el Católico y el Emperador Maximiliano I de Austria. Dentro de este conjunto sobresalen las armaduras de Carlos V y de Felipe II como conjunto de mayor importancia y núcleo sobre el que 
se fundamenta el resto de la Colección. Junto con él destacaban otros conjuntos significativos del proceso de formación de la Colección actual, a pesar del incremento irregular de sus fondos desde el siglo XVI al XIX: entre ellos se encuentran las armas medievales procedentes del Tesoro del Alcázar de Segovia; las armas de fuego de Carlos V y Felipe II; las armaduras de los Príncipes e Infantes de España siendo niños; los trofeos militares; y los regalos diplomáticos y familiares como los enviados por las siguientes personas: el Duque de Mantua a Carlos V; el shogun Toyotomi Hideyosi a Felipe II; Carlo Manuel I de Saboya y Jacobo I de Inglaterrra a Felipe III; la Infanta Isabel Clara Eugenia y el Cardenal Infante Don Fernando a Felipe IV; o el sultán de Turquía a Carlos III entre otros. El último conjunto de especial importancia dentro de la Colección, lo constituyen las armas de fuego forjadas en Madrid para las actividades venatorias de la corte, de gran reputación en todo el continente.

La riqueza y variedad de los fondos de la Colección permiten también diversas lecturas de la misma ya que, a través de sus fondos, se puede ilustrar, parcialmente, la Historia de España de un periodo, el contexto cortesano para el que fueron creados, y la evolución decorativa, tipológica $\mathrm{y}$ funcional de las armas de lujo para la Corte española desde el Renacimiento hasta el siglo $\mathrm{XIX}^{2}$.

\section{Los fondos medievales. El reinado de los Reyes Católicos y la introducción de la casa de Austria}

Los fondos medievales y de transición al Renacimiento, constituyen un conjunto de gran importancia por su significado, a pesar de su exiguo número y su diversa procedencia. Una parte se encontraba en la armería de Carlos V, quien había heredado las armas de su padre, de sus abuelos y de algunos de sus contemporáneos. Un segundo conjunto procede del Tesoro Real del Alcázar de Segovia, trasladado a la Armería de Madrid por orden de Felipe II. Un tercer grupo está compuesto por diversas compras, donaciones y traslados de preseas reales efectuadas entre los reinados de Fernando VII hasta Alfonso XII. Destaca la representación de los emblemas de los Reinos de Castilla, León y Aragón, presentes en los acicates y el manto de Fernando III, procedentes de su enterramiento de la Catedral de Sevilla, y en la cimera del Drac Alat atribuída a Martín I el Humano. Junto con ellos sobresalen el estoque real de los Reyes Católicos, utilizado como espada de ceremonia en la Corte Española hasta el siglo XVIII. 
El reinado de los Reyes Católicos y el armamento de fines del medievo, también está representado por armas de diversa procedencia que enmarcan la actividad desarrollada en este periodo. Se conservan armas de guerra contemporáneas a la toma de Granada, compuestas por piezas defensivas ilustrativas de los talleres españoles, italianos y alemanes; así como dos de las armas de fuego portátiles más antiguas conocidas en España, aún deudoras en ciertos aspectos, de la ballestería que suplantarán con el tiempo. Dentro de este grupo merecen especial mención los capacetes y las piezas de armadura asociados a una peculiar producción peninsular de gran prestigio, cuyos talleres no han sido aún identificados, aunque se suponen de procedencia aragonesa. El propio sultanato nazarí está presente mediante una exigua pero importante muestra de su panoplia, ya que se conserva un ejemplar de cada uno de los tres tipos de armas de creación granadina: una jineta procedente de la colección del Cardenal Infante Don Fernando; una adarga de cuero conservada en la armería de Carlos V; y una daga de orejas asociada a un cinturón con escarcela y una funda para un Corán, estos últimos capturados en la batalla de Lucena a Muhammmad XII, Boabdil, y presentados a Alfonso XIII por el Marqués de Viana como parte del legado Villaseca.

La Colección conserva armas relacionadas con algunos de los hechos y personajes destacados del Reinado de los Reyes Católicos por medio de sus armas, como es el caso de la espada de Gonzalo Fernández de Córdoba, el Gran Capitán, o las armas del Emperador Maximiliano I de Austria, concretamente, una testera de caballo, una coracina y dos bardas. Las primeras aluden a su poder y dinastía por medio de una decoración de carácter heráldico y alegórico en la que priman los emblemas imperiales. Estas armas proceden de la colección de Carlos V, donde también se custodiaban las armas de su padre, el Rey Felipe I de Castilla, el Hermoso, representativas de las alianzas matrimoniales entre los Reyes Católicos y Maximiliano. La armería de Felipe el Hermoso se encuentra actualmente dividida entre la Hofjagd und Rüstkammer de Viena (Kusthistorisches $\mathrm{Mu}$ seum) y la Real Armería de Madrid, donde fundamentalmente se encuentran las armas utilizadas desde su matrimonio con Juana de Castilla, entre las que destacan, una espada de dos manos con su mote personal, y celadas y testeras de procedencia flamenca, alemana e italiana; entre éstas, dos celadas del taller milanés de Negroli, y tres armaduras que responden a dos ejemplos únicos en la producción flamenca y española del periodo. 


\section{Las Armerías de Carlos V y Felipe II}

Las armerías de Carlos V y Felipe II constituyen el núcleo fundamental de la Colección, sobre todo en lo que respecta a la armería imperial. Por su parte las armas de Felipe II no pueden estar disociadas de las de su padre, dadas las estrechas relaciones existentes entre ambas, y por su procedencia alemana o italiana dentro de un mismo periodo cronológico. De hecho, la mayor parte de las armaduras de Felipe II fueron forjadas en vida de Carlos V, coincidiendo, en ocasiones, en su elaboración con las del Emperador. El conjunto de las armaduras de Carlos V y Felipe II fue forjado entre 1519 y 1560, en pleno Renacimiento, durante el momento de esplendor del arte de la armadura.

La complejidad y la gran riqueza de ambas armerías impide abordar una panorámica completa, aunque fuera de manera elemental. Por ello nos limitaremos a tratar algunos de sus rasgos y componentes fundamentales en forma de somera introducción a las mismas. El primer concepto a tener en cuenta, es que nos encontramos ante una Colección de armas cuyo uso debe ser encuadrado en el mundo cortesano contemporáneo. No se trata, esencialmente, de un conjunto de armas de guerra, sino de una colección de armas de lujo como representación del poder, destinadas a ser usadas en los diferentes eventos de la Corte: justas, torneos, paradas militares, juegos de cañas, etc. La mayor parte de las armaduras de Carlos V y Felipe II fueron forjadas siguiendo el concepto de guarnición de armadura inventado por Maximiliano I: consistió en una armadura de base dotada de piezas de refuerzo o complemento, decoradas uniformemente, de manera que el intercambio de las distintas piezas que la componen, podía dar lugar a la formación de diversas figuras destinadas al combate a pie, a la justa y al torneo ecuestre, a la guerra en sus diferentes variantes o a la parada. La Colección Real española sobresale, entre otras colecciones afines, por haber conservado gran parte de estas piezas auxiliares y un alto número de bardas o armaduras de caballo, asociadas, en algunos casos, a dichas armaduras.

En este tipo de armas se valoraba especialmente la perfección técnica y constructiva, el diseño formal y la decoración, siendo esta última rica en connotaciones de todo tipo, desde lo caballeresco hasta el reflejo de las ideas y los temas del Humanismo mediante motivos alegóricos, religiosos, heráldicos y/o dinásticos, o recreando la tradición clásica de época romana ${ }^{3}$. La ejecución material de la decoración se realizaba mediante técnicas destinadas a mostrar o realzar la belleza y riqueza 


\section{La Real Armeria de Madrid}

de las piezas, gracias a superficies grabadas al agua fuerte, leves repujados, superficies doradas o plateadas, pavonados, ataujía de oro y plata, etc. Por ello en toda Europa las armas de lujo sólo podían ser forjadas y decoradas en un limitado número de talleres altamente especializados. Los más importantes estaban situados, por razones históricas y geográficas, en Alemania y en el norte de Italia. En el caso de Carlos V destacan los talleres de Kolman y Desiderius Helmschmid de Augsburgo, y de Filippo Negroli y hermanos de Milán. En el caso de Felipe II, Franz y Wolfgang Grosschedel de Landshut, Desiderius Helsmchmid y Antón Peffenhauser de Augsburgo. Estos armeros son objeto de una predilección especial, pero tanto Carlos V como Felipe II poseyeron armas procedentes de otros talleres de alto nivel, como el de Mattheus Frawenbrys, Caremolo Mondrone, o Bartolomeo Campi, bien por ser encargos concretos, o por haber sido piezas presentadas por diversos personajes relacionados con la Corte Española ${ }^{4}$. En cualquier caso, es de gran importancia resaltar que estos talleres se encontraban situados en territorios dependientes de la Monarquía Española, a la que dedicaron lo mejor de su producción. La aristocracia española, austríaca e italiana siguió en parte el gusto de Carlos V y de Felipe II. Los cambios en los gustos personales y las circunstancias históricas condicionaron el auge o decadencia de los diferentes centros productores. Es el caso de la decisión de Felipe II de primar la producción de Landshut en detrimento de Augsburgo, o del declive de los centros alemanes y la consolidación de los italianos, entre otros motivos, por la pérdida de poder específico en Alemania de la Monarquía Hispana. Alemania continuó siendo un centro de gran importancia, pero no recuperó el prestigio que disfrutó gracias al patronato de la Corona de España.

Entre las armas de Carlos V y Felipe II también destaca un reducido, pero importante, conjunto de armas de fuego compuesto por pistolas y arcabuces que responden a los primeros modelos de armas de rueda conocidas. Estas pistolas pueden ser lisas o decoradas con placas o piezas de hueso embutidas. Al igual que en el caso de las armaduras proceden de centros armeros alemanes e italianos, con especial mención a los arcabuceros Peter Pech de Munich, y a la familia Marcuarte originaria de Augsburgo. Cabe reseñar, también, un pedreñal considerado como una de las primeras armas conocidas de la producción catalana de Ripoll.

Entre las armas blancas destacan el estoque imperial y diversas espadas a juego con las armaduras, para acompañar al traje civil o destinadas a la caza. Relacionadas con esta última actividad, funda- 


\section{Alvaro Soler del Campo}

mental en el mundo cortesano contemporáneo, se conservan diversas ballestas y saetas a la moda española para caza mayor y volatería; dos cerbatanas con sus fundas pertenecientes a Felipe II; y una excepcional armadura para lebrel, incompleta, procedente de la armería de Carlos $\mathrm{V}$ y destinada sin duda a un animal muy apreciado: se trata de dos piezas metálicas rígidas para los flancos, con decoración grabada alusiva a la caza del jabalí que indica su verdadera finalidad.

Las armerías de Carlos V y Felipe II, al igual que las de sus sucesores, muestran, parcialmente, el contexto cortesano y político en el que se inscriben. En ellas se custodiaban armas de gran importancia simbólica para reflejar el poder de la dinastía, por ejemplo los trofeos procedentes de las principales campañas militares como Pavía, Mühberg y Lepanto. También custodiaban bellas armas procedentes de regalos por motivos familiares y/o diplomáticos: son los casos de los regalos enviados en 1534 y 1536 por el Duque de Mantua a Carlos V; la armadura a la romana de Bartolomeo Campi regalada por el Duque de Urbino a Felipe II; o las armas japonesas enviadas por el shogun Toyotomi Hideyosi y presentadas a Felipe II en 1584, regalo en el que, sobre todo, debió pesar su condición de Rey de Portugal.

Mención especial merecen las espadas o estoques benditos consagradas el día de Navidad por los Pontífices romanos, en señal de amor a los príncipes más escogidos de la Cristiandad, para que acrecentasen la fe católica y defedieran la sede apostólica. El estoque significaba la infinita potencia de Dios y la soberana potestad temporal de su vicario en la tierra. La Real Armería conserva el mayor conjunto preservado, compuesto por nueve estoques pontificios, a pesar de que la mayor parte de éstos no han llegado completos hasta nuestros días. El conjunto abarca, desde el enviado por Eugenio IV a Juan II en 1446, hasta el regalado por Paulo V a Felipe IV en 1618.

La Corte está representada por las armas asociadas a personajes como Juan de Austria, Hernán Cortés, Alejandro Farnesio, Francisco de Pizarro o Hernán Cortés, estos últimos presentes gracias a ingresos de época más reciente.

\section{Felipe III}

Los fondos de la Real Armería datados en el siglo XVII se caracterizan por la desigual importancia de los conjuntos en los que se dividen. Constituyen, además, una sección de la Colección que ha sido eclipsada 


\section{La Real Armeria de Madrid}

por la fama, en todos los aspectos, de las armerías de Carlos V y Felipe II, pero en realidad debe ser considerada como uno de los conjuntos más importantes existentes sobre el periodo, con obras de gran calidad o importancia histórica y artística, que merecen una consideración diferente a la que tradicionalmente se le ha dado. Ello es, en parte, debido a una feliz concurrencia de diversos ingresos en la Colección, que continuó enriqueciéndose con armas pertenecientes a la Familia Real y con regalos diplomáticos, que constituyen un conjunto de gran interés representativo del armamento de lujo procedente de diversos centros europeos. Esta época marca un momento de transición y supone un cambio importante en la historia del arte de la armadura. La producción de armas de lujo contempla, a principios de siglo, el ocaso del manierismo para dar paso a los nuevos usos y modas que se impusieron a raíz de la guerra de los Treinta Años. Paradójicamente, cuando las armas de fuego se impusieron sobre las armaduras, éstas recuperaron el principio de funcionalidad, con arneses para la guerra sencillos y prácticos destinados a la nueva caballería que dominó los campos de batalla.

En el reinado de Felipe III pueden diferenciarse cuatro grupos, claramente definidos, que hacen de este conjunto uno de los más ricos del periodo. El primero está compuesto por las armas encargadas para su servicio y el de sus hijos, entre las que destaca la peculiar producción de Eugui, centro creado en 1595 por Felipe II en las cercanías de Pamplona con armeros milaneses, con el fin de dotar a la Corte Española de un centro de producción propio ${ }^{5}$. Eugui no fue, sin embargo, efectivo hasta el reinado de Felipe III, durante el que sobrevivió un corto y agitado periodo de tiempo: su producción es, lógicamente, deudora de su origen milanés, pero destaca por estar dotada de una personalidad propia, por su elegancia y por la alta calidad técnica y decorativa de sus piezas, como muestran las dos armaduras conservadas de Felipe III; las pertenecientes al Príncipe Felipe, futuro Felipe IV; las de los Infantes Don Carlos y Don Fernando siendo niños; un arcabuz regalado a Felipe IV, Príncipe; y un juego de borgoñota y rodela actualmente incompleto. Las armaduras de niño destinadas a los Infantes y las propias armaduras regaladas a Felipe III niño por los Duques de Terranova y Saboya, constituyen uno de los conjuntos más destacados de la Colección, completado con otras armaduras infantiles de procedencia incierta y una exquisita guarnición posterior para Don Baltasar Carlos. Las armaduras infantiles no deben ser consideradas como un juguete, sino como armas dotadas de un alto valor de representación al igual que las destinadas a los adultos. 
Un segundo grupo está compuesto por las armas compradas por el Rey para enriquecimiento de la Colección. Se componen por un rico juego de parada y armas de asta adquiridos a Andrés de Loidi de San Sebastián. Junto con él destaca la compra de un importante lote de ballestas de caza, rodelas, y armas blancas y de fuego al Licenciado Ramírez del Prado.

El tercer grupo está integrado por regalos propios de las relaciones diplomáticas y familiares, no siempre diferenciadas con claridad. En él también se podrían haber incluido las armaduras de niño presentadas por los Duques de Saboya y Terranova, contenidas en el primer apartado sólo por razones de conveniencia formal en tanto que son armaduras de niño. Destacan por su significación los regalos enviados en 1604 y 1614 por Jacobo I de Inglaterra, compuestos por armas de fuego y ballestería, ricamente decoradas, en las que sobresalen las llaves snaphance de diseño inglés ${ }^{6}$. Dentro de este grupo también podría considerarse un pequeño conjunto incompleto de armas de parada compuesto por una gola y jaeces para caballería datados hacia 1604-1621, y asociados al Archiduque Alberto de Austria. Aunque se desconoce su procedencia, la atribución de propiedad al Archiduque se debe a que, el dorso de la gola, representa el asedio de Ostende mantenido por sus tropas entre 1601 y 1604, con el victorioso resultado de la toma de la plaza. El frente de la gola destaca por una magistral composición del espacio que permite la respresentación minuciosa, muy dinámica, de diversos cuerpos de ejército sobre un paisaje relacionado con la batalla de las Dunas. La toma de Ostende, cuya planta fue representada en la gola con gran fidelidad, ha sido considerada como la victoria más importante del Archiduque.

Dentro de la armería de Felipe III, y del resto de la Colección en general, brilla con luz propia el suntuoso regalo enviado, en 1603, por su cuñado el Duque Carlo Manuel I de Saboya con motivo de la venida a la Corte madrileña de sus tres hijos ${ }^{7}$. El regalo, espléndido, se conserva en su mayor parte en la Real Armería, salvo algunas piezas de armadura con las que fue enterrado el Príncipe Don Carlos en El Escorial, y un pequeño lote de piezas en colecciones francesas e inglesas. En la actualidad se conservan una armadura ecuestre de parada con dos bardas a juego, dos celadas a la oriental con sus respectivas golas, dos escudos de parada y un sable ricamente guarnecido. Curiosamente pueden ser datadas hacia 1585, por lo que se ha considerado el carácter anticuario del regalo. Una de las bardas ha sido felizmente recuperada por el Patrimonio Nacional en fecha reciente tras haber sido robada de la Real Armería, en 1838, y subastada en la venta Oxenham de Londres en 1843. 


\section{La Real Armeria de Madrid}

La armería de Felipe III custodiaba, por tanto, obras de procedencia española, inglesas e italianas en virtud de los encargos personales y de los regalos recibidos. El contexto político del reinado supuso el predominio de los centros italianos en la comisión de armaduras para la Corte, sobre todo las procedentes de Milán dada la tradición armera de la ciudad y su importancia política para la Corona Española. Esta circunstancia se ilustra claramente en un cuarto grupo compuesto por las armaduras pertenecientes a diversos personajes de la Corte, que realzan la preponderancia de Milán en la comisión de este tipo de objetos, y constituyen, a la postre, un núcleo muy significativo de los principales talleres activos a finales del siglo XVI e inicios del XVII. Son los casos de las armaduras de Don Antonio de Zúniga y Velasco, quinto Conde de Nieva, y de Don Juan Fernández Pacheco y Acuña, quinto Duque de Escalona; esta última, obra de Pompeo della Cesa datada hacia 1595. En este círculo también se deben destacar las forjadas por el Maestro del Castello para Emanuel Filiberto de Saboya, hacia 1606, y para Don Pedro Franqueza, Conde de Vilalonga y Secretario de Estado de Felipe III, hacia 1610, que debió pertenecer con anterioridad a D. Pedro Enriquez de Acevedo, Conde de Fuentes y ex gobernador de Milán.

\section{Felipe IV}

Durante el reinado de Felipe IV las armaduras perdieron toda la importancia que habían tenido en épocas anteriores. Durante la primera mitad del siglo continuaron siendo valiosos objetos de representación, pero el perfeccionamiento técnico de las armas de fuego provocó su desaparición a lo largo de la centuria. De este último momento de esplendor del arte de la armadura, datan dos valiosos regalos enviados, respectivamente, por la Infanta Isabel Clara Eugenia a su sobrino Felipe IV, en 1624 y 1626, y por el Cardenal Infante Don Fernando siendo gobernador de Milán.

Los regalos de 1624 y 1626 fueron enviados cuando Felipe IV contaba diecinueve y veintiún años ${ }^{8}$. Consistían en dos conjuntos de armaduras conservados, en mayor o menor medida, incompletos en la Real Armería de Madrid. Los elementos restantes, o bien han desaparecido, o se encuentran dispersos en colecciones europeas y americanas. Los regalos se produjeron, paradójicamente, en un momento en el que las armaduras comenzaban a perder la importancia que habían tenido en épocas anteriores, debido en gran parte, al avance de las armas de fuego. En 
la primera mitad del siglo XVII tuvieron lugar las últimas producciones continentales de importancia. En ellas se volvió a valorar, por lo general, la funcionalidad y sencillez de las primeras armaduras, sin que supusiera renunciar a guarniciones ricamente decoradas. Los regalos de Isabel Clara Eugenia destacan dentro de este contexto por su opulencia y sofisticación, tanto desde el punto de vista técnico como estético, a pesar de tratarse de armaduras concebidas, funcionalmente, para la guerra. Constituyen uno de los conjuntos más importantes conservados del último periodo de importancia en la historia del arte de la armadura.

El primer regalo constaba de dos guarniciones compuestas cada una por dos armaduras con sus complementos, obra de un armero flamenco anónimo, de probable origen francés, conocido como el «maestro del MP» en referencia a su marca: un escudete coronado con tres flores de lis en el campo, flanqueado por las iniciales M/P. Las dos guarniciones responden a una misma concepción al estar compuestas por sendas armaduras de guerra completadas con testeras de caballos que, básicamente, reproducen un mismo número y tipología de piezas. Las celadas destacan por tener en la vista un monograma calado con el nombre «Ysabel» bajo corona real. Este monograma se ha interpretado, tradicionalmente, como una alusión a Isabel Clara Eugenia o a Isabel de Borbón, pero probablemente aluda a esta última por ser la esposa de Felipe IV y por estar asociado a la Corona Real.

El regalo de 1626 también estaba compuesto por cuatro armaduras con algunos elementos de complemento o refuerzo. Se diferencian del anterior porque no se dividen en dos guarniciones, sino que cada una de estas armaduras está decorada de diferente manera, es decir, no son guarniciones compuestas por más de una armadura decorada a juego. Tipológicamente guardan, sin embargo, una analogía clara con el regalo de 1624, tanto desde el punto de vista formal como conceptual, ya que también se trata de cuatro armaduras de guerra, dos de ellas específicas de infantería. Las armaduras de este regalo destacan y se diferencian del anterior por una rica y sofisticada decoración basada en follajes grabados de distintas hechuras, inscritos o no por patrones geométricos, que ocupan toda la superficie de las piezas. Ninguna de las piezas del regalo de 1626 conservadas en la Real Armería muestra la marca del maestro del MP, por lo que la atribución de su autoría no está documentada. Salvo la cuarta armadura del regalo, las tres primeras se conservan en la Real Armería muy incompletas, por la determinación de Felipe IV de regalar dos de ellas a Don Juan José 


\section{La Real Armeria de Madrid}

de Austria y al margrave Federico Darmstadt. Por su parte, la tercera armadura del regalo fue entregada a Don Juan José de Austria con todas las piezas que tenía en 1647. Se ha señalado que la entrega de estas armas, en 1647, coincide con la salida hacia Nápoles de Don Juan José cuando contaba dieciocho años, un año antes de que Juan Melchor Pérez realizara, en 1648, el busto de bronce del joven general conservado en el Museo del Prado.

El regalo del Cardenal Infante Don Fernando fue enviado entre 1633 y 1634. Consistió en una rica armadura milanesa pavonada guarnecida con medallas de plata, una armadura de caballería, y una exquisita armadura, también de caballería, para el Príncipe Baltasar Carlos siendo un niño de cuatro o cinco años. Las dos últimas estaban decoradas de manera análoga por haber tenido o conservado superficies pavonadas lisas y estrechas bandas doradas siguiendo los bordes de las piezas.

Junto con estas armaduras destinadas al servicio real es digno de reseñar un pequeño grupo de armaduras de asedio a prueba de arcabuz que, históricamente, ha venido pasando desapercibido por haber estado disperso entre los fondos de la Colección.

Tras la muerte del Cardenal Infante en 1643, ingresó en la la Real Armería una parte de sus armas, entre las que se conservan una rodela milanesa a prueba de arcabuz, y una pistola de Brescia firmada por Angelo Lazarino Cominazzo. En la Real Armería ingresaban también las armas dotadas de una especial significación, como los trofeos militares por ser objetos relevantes para mayor prestigio de la Corona Española. Entre ellos se encuentra una espada de caballería de Wilhem Wirsberg de Solingen, tomada, en 1634, a Bernardo, Duque de Weimar, durante la batalla de Norlinga, siendo General en Jefe del ejército sueco. Los ejércitos al mando del Cardenal-Infante Don Fernando, y las fuerzas del Imperio y Baviera mandadas por Fernando de Hungría y el general Matthias Gallas, derrotaron a los suecos y a los sajones mandados por Gustav Horn y Bernardo de Weimar.

La desaparición de las armaduras durante el siglo XVII centró la producción europea de armas de lujo en las armas blancas, y, especialmente, en las de fuego, cuya producción había crecido de forma notable en todo el continente desde mediados del siglo XVI. Con todo, este tipo de armas no debía perder la alta calidad técnica y artística exigida a cualquier objeto de lujo. En la primera mitad del siglo XVII se incrementa, notablemente, el número de fondos relativos a armas de fuego y armas blancas. Entre las primeras cabe destacar un claro predominio de las pistolas y arcabuces de procedencia italiana, sobre 
todo de Brescia, con firmas tan prestigiosas como las de la familia Cominazzo o la de Giovanni Battista Francino. La producción española está representada por dos arcabuces de Felipe IV siendo Príncipe; el primero de claro influjo italiano por proceder de Eugui; y el segundo con llave firmada por Juan Salado y cañón procedente de Brescia.

Los reinados de Felipe III y Felipe IV han supuesto una notable aportación a la colección de armas blancas. Destacan las forjadas por espaderos toledanos, a pesar de no haber conservado, por lo general, sus empuñaduras originales, y de ser un centro productor cuya historia necesita ser revisada, ya que sólo está claramente documentado, como centro de importancia continental, desde el segundo tercio del siglo XVI hasta el segundo cuarto del siglo XVII. Toledo está representado en la Real Armería con firmas de prestigio como las de Sebastián Hernández, Hortuño de Aguirre, Tomás de Ayala o Pedro Belmonte.

En el contexto europeo merecen mención especial una espada con cazoleta decorada según un grabado de Antonio Tempesta, y la producción de Solingen, como centro competidor de Toledo, representado en la Colección por un número reducido de espadas que, sin embargo, ostentan firmas como las de Clemens Kuller, Jacob y Clemens Brach, Johan Tesche o Wilhem Wirsberg para la ya citada espada del Duque de Weimar.

Curiosamente, la segunda mitad del siglo XVII, coincidente en su mayor parte con el reinado de Carlos II, es el único periodo del que casi no existe representación, casi interrumpiendo la continuidad cronológica y dinástica en la historia de la Colección desde el reinado de los Reyes Católicos. Con el mencionado reinado se relacionan un arcabuz y una espada de supuesta atribución a Carlos II.

\section{El siglo XVIII. La Casa de Borbón}

La Guerra de Sucesión, y por tanto el cambio dinástico, es representado, testimonialmente, por dos moharras de estandarte de Carlos VI. La subida al trono de Felipe V en 1700 abre un segundo periodo en la historia de la Real Armería. En primer lugar, la definitiva desaparición de las armaduras cambiará el carácter de la Colección que, desde entonces, se compondrá, fundamentalmente, por armas de fuego. En segundo lugar, el cambio de época, las nuevas circunstancias políticas y las modas francesas promovidas por la Casa de Borbón, propiciaron un cambio en el gusto de la Corte, pero se mantuvieron los encargos 
a Italia, que todavía se encontraba dentro del ámbito de influencia de la Corona Española.

Los ingresos en la Colección continúan estando relacionados con el servicio de la Familia Real y la acción exterior de la Monarquía, bien por medio de trofeos militares con objetos de prestigio, como la indumentaria, las armas blancas y las armas de fuego capturadas en la toma de Orán, de 1732, por Felipe V, o bien por medio de regalos diplomáticos. Entre estos últimos destacan las escopetas recibidas por Carlos III del sultán de Marruecos, en 1766; del sultán de Turquía, en 1787, algunas de ellas guarnecidas con llaves catalanas de Ripoll; las escopetas presentadas por el Bey de Argel, en 1770; y las pistolas recibidas por Carlos IV del Bey de Túnez. En su mayor parte se corresponden con espingardas decoradas según los patrones extendidos por gran parte de los territorios islámicos en esta época, que pervivirán, en gran medida, durante todo el siglo XIX, es decir, decoración profusa según diversas combinaciones de cañones damasquinados, cajas con piezas gutiformes de coral, marfil, nácar o carey embutidas, y chapas y abrazaderas de plata grabadas o pedrería.

Durante el reinado de Carlos II los fabricantes de armas de fuego españoles habían adquirido un profundo conocimiento de la tecnología y la decoración francesas relativas a la fabricación de armas de fuego. Sobre esta base, la llegada al trono de Felipe V propició el desarrollo de una manufactura de armas de fuego de lujo en Madrid, destinada a satisfacer las necesidades cinegéticas de la Corte. En este sentido recordamos el importante papel de la caza en la vida cortesana, especialmente en Madrid, villa elegida desde el siglo XVI como sede de la Corte, entre otras razones, por sus posibilidades cinegéticas. Madrid se convirtió en uno de los principales centros europeos dedicados a la fabricación de armas de lujo. La arcabucería madrileña constituye un hito dentro de las artes industriales y decorativas españolas, por la gran calidad técnica de sus cañones y por la riqueza ornamental de los ejemplares más señalados. Su producción fue, sin embargo, limitada, ya que los arcabuceros madrileños centraban su carrera con la esperanza de trabajar para el servicio real.

La reputación internacional de las escopetas madrileñas del siglo XVIII estaba basada en la belleza del conjunto del arma, en la fiabilidad de sus llaves o mecanismos de ignición, y en la calidad de los cañones, motivo de falsificación en el resto de Europa. Las escopetas madrileñas se caracterizaban por montar cañones pavonados decorados con motivos vegetales, geométricos o figurativos en oro, acompañados por la firma y marcas del arcabucero. Las llaves seguían la tradición española, o 
la moda francesa, y solían estar ricamente grabadas y doradas albergando motivos mitológicos, alegóricos o cinegéticos. La caja podía estar puntualmente tallada o embellecida con alambre de acero o de plata, mientras que sus guarniciones metálicas, como la culata o el guardamonte, eran decoradas a juego con la llave.

El conocimiento de esta manufactura es limitado a pesar de su importancia. No existen tratados contemporáneos que expliquen los sistemas de forja, o que recojan el testimonio de los arcabuceros más conocidos en cuanto a técnicas constructivas y decorativas. Sólo se conoce el famoso Compendio de los arcabuceros de Madrid, de Isidro Soler, publicado en 1795, cuando esta industria ya se encontraba en su ocaso. En ella se hace breve reseña del inicio de la arcabucería madrileña, de los sistemas de forja y de los principales arcabuceros habidos hasta su tiempo.

La elaboración de estas escopetas para el servicio real recaía en el arcabucero del Rey, título que sólo alcanzaban los mejores artesanos, tras superar pruebas de maestría y presentar un arma de excepcional calidad que atestiguara su valía. El arcabucero tenía una serie de privilegios, como disponer de uniforme y de una pensión, que le permitía trabajar en exclusiva para el Rey. Su trabajo era atestiguado con marcas localizadas en la recámara de los cañones y sobre las platinas de las llaves; los punzones empleados eran custodiados en la Real Armería para evitar usos indebidos. Desde un punto de vista decorativo se distinguen dos tipos de arcabuces: unos modelos muy sencillos sin decoración, eminentemente funcionales; y otros, mucho más elaborados de aparato, muy escasos y que, posiblemente, correspondan a encargos especiales, o a las armas que el arcabucero debía hacer para mostrar su valía cuando entraba al servicio del Rey.

Generalmente era el propio arcabucero el encargado de la decoración del arma, pero se conoce un caso en el que se solicita el concurso de un profesor de la Real Academia de Bellas Artes, concretamete, la colaboración de Jacques Lavau y Salvador Cenarro para un juego de escopeta y dos pistolas destinadas a Carlos III. La decoración de las escopetas madrileñas es tributaria de los repertorios de diseños publicados en París a finales del siglo XVII e inicios del siglo XVIII, como los de Claude Simonin, Laurent Le Languedc, DeLacollombe y Demarteau. El fin de Madrid no es bien conocido, pero a juzgar por Soler, ya estaba en decadencia a finales del siglo XVIII. Es posible que el golpe de gracia a esta decadencia lo ejecutara la propia invasión napoleónica, pero nada se puede asegurar. En cualquier caso, parece ser que las antiguas técnicas perduran, al menos, hasta el segundo 


\section{La Real Armeria de Madrid}

tercio del siglo XIX, según lo atestigua una escopeta de caza de Francisco Lopez fechada en 1833, y recientemente adquirida por el Patrimonio Nacional: híbrido entre la modernidad y la tradición de la escuela, por el cañón en dos órdenes, con abrazaderas y damasquinado al uso.

Junto con la arcabucería madrileña, la Real Armería conserva un conjunto de armas de fuego contemporáneas de procedencia italiana, francesa, portuguesa y alemana, entre las que se encuentran ejemplares de interés técnico por tratarse de armas de aire comprimido y repetición. Sobresalen dos escopetas de la Real Fábrica de Nápoles, manufactura establecida en 1757 por el futuro Carlos III de España, firmadas y fechadas por Michele Battista en 1772, considerado como la principal figura de esta manufactura.

Los años finales el siglo XVIII son importantes para la historia de la Colección ya que, en 1793, se lleva a cabo un inventario general de la misma, que fue publicado por Ignacio de Abadía en Madrid bajo el título, Resumen sacado del inventario general histórico que se hizo en el año de 1793 de los arneses antiguos, armas blancas y de fuego, con otros efectos, de la Real Armería. Dicho título constituye la primera publicación de un catálogo de la Colección, a pesar de ser una versión resumida del inventario realizado el citado año.

\section{El siglo $\mathrm{XIX}$}

Uno de los hechos más destacados del siglo XIX en España, es el declive de Madrid como centro productor, dando paso al auge de los centros vascos de Eibar y Placencia de las Armas, situados en una región dedicada a la manufactura de armas de guerra desde la Edad Media. La nueva situación se reflejará en la Colección Real por la representación de la armería vasca a lo largo del todo el siglo.

Entre las armas conservadas del reinado de Fernando VII, destacan un par de pistolas de la fábrica de Ybarzabal de Eibar, y los regalos realizados por el Conde de la Fernandina y la Provincia de Guipuzcoa, consistentes, respectivamente, en una rica escopeta bresciana de Frachetti e Minelli, y un juego de pistolas eibarrés de Juan Esteban Bustindui y Juan Andrés de Mendizábal.

En el reinado de Isabel II la comisión de armas a los talleres vascos de Eibar y Placencia de las Armas se compagina con las producciones de la Fábrica de Armas de Toledo, tanto en lo relativo a las armas de gala, como espadines, como en lo referente a las armas 
destinadas a la Guardia Real. Lo más destacado es, sin embargo, el trabajo desarrollado en el seno de la Colección, bajo la condición de Armeros Reales, por Plácido y Eusebio Zuloaga. Entre las actividades, no siempre afortunadas, de estos armeros, se debe reseñar la labor de estudio y reinterpretación de la panoplia renacentista, con nuevas creaciones para la Corte, así como otras armas para el uso personal de Isabel II o de Francisco de Asís.

Los reinados de Alfonso XII y Alfonso XIII se caracterizan por el ingreso, casi exclusivo, de armas de fuego de caza y armas blancas de gala para su servicio. Las armas conservadas demuestran una gran variedad en los encargos por estar representadas las principales firmas europeas del momento.

Uno de los hechos más destacados del siglo XIX fue la publicación de las principales obras documentales y catalográficas existentes sobre la Colección, con especial mención a la labor de Achille Jubinal y Gaspard Sensi, (La Armeria Real ou collection des principales pièces de la galerie darmes anciennes de Madrid), Martinez del Romero (Catálogo de la Real Armería mandado formar por S.M. siendo director general de la reales caballerizas, armería y yeguada, el Excmo. Señor Don José María Marchesi. Madrid, 1849); y del Conde Viudo de Valencia de Don Juan.

En 1884 un incendio destruyó, parcialmente, la Armería construída por Felipe II. Sin reparar en gastos Alfonso XII, ordenó la construcción de un nuevo edificio que constituye su sede actual. La temprana desaparición del Monarca impidió que viera culminada su obra, que se terminó finalmente, por voluntad de la Reina Doña María Cristina de Habsburgo. La planta noble del nuevo edificio fue concebida como una gran sala de armas decorada con tapices y panoplias en la que se instaló toda la Colección. La nueva instalación, inagurada en 1893, marcó un hito, a todos los efectos, en la propia historia de la Colección, condicionando, desde entonces, hasta el presente la imagen de la misma.

\section{Notas}

1 Sobre las circunstancias que rodearon el traslado de la Coleccción a Madrid y su instalación en la nueva armería ver: SOLER DEL CAMPO, A. (1998): «La armería de Felipe II". Reales Sitios 135, 24-37.

2 En 1898 se publicó el último catálogo general de la Colección por el Conde Viudo de Valencia de Don Juan. Este catálogo está, en gran parte, superado científicamente, pero sigue plenamente vigente como guía de referencia para el estudio de la misma. En la fortuna crítica de este trabajo no se detallarán cada una de las 


\section{La Real Armeria de Madrid}

referencias específicas de las armas y conjuntos aquí citados con el fin de no hacerla demasiado prolija. Su consulta no plantea problemas utilizando el índice de la obra. Valencia DE DON JuAN, Conde Vdo. De. (1898): Catálogo Histórico-Descriptivo de la Real Armería de Madrid. Madrid.

3 Sobre los aspectos simbólicos de las armas y sobre todo su consideración como obras de arte de alto nivel ver: PYHRR, S.W. y GODOY, J.A. (1998): Heroic Armor of the Italian Renaissance. Filippo Negroli and his Contemporaries (With essays and a compilaton of documents by Silvio Leydi). Nueva York. The Metropolitan Museum of Art. 8 Oct. 1988 - 17 Jan. 1999. Para las armas dentro del contexto de los juegos caballeresos: RANGSTRÖM, L. [Ed.]. (1992): Riddarlek och Tornerspel. Sverige-EuropaTournaments and the Dream of Chivalry. Stockholm. Livrustkammaren Stockholm 12.6.1992-6.12.1992.

4 Como introducción a la obra de los principales armeros que ponen su arte al servicio de Carlos V y/o Felipe II, y el contexto en el que éste se desarrolla: BoccIA, L.G.y Coelho, E.T. (1967): L' Arte dell'armatura in Italia. Milán. Cortes Echanove, J.(1963): Armas y armeros en la época de Felipe II. El Escorial (1563-1963). Historia. Literatura (Catálogo de la exposición.). Madrid, 257-292. LAVIN, J. D. (1965): A History of Spanish Firearms. Nueva York. Reitzenstein, A. (1954): Die Landshutter Plattner Wolfgang und Franz Grosschedel. Münchner Jahrbuch der Bildenden Kunst, 142-153. Reitzenstein, A, (1971): Antoni Peffenhauser. Waffen und Kostümkunde, 111-127. THOMAS, B. (1944): Deutsche Plattnerkunst. München. THOMAS, B. (1980): Augsburger Harnische und stangenwaffen (Plattner, Ätzmaler, Goldschmiede). Welt im Umbruch. Augsburg zwischen Renaissance und Barock, vol.II. Rathaus, Augsburg 72-92, 502-537. Thomas, B. y Gamber, O. (1958): “L'Arte milanese dell'armatura». Storia di Milano. Milán. Vol. XI. Il declino spagnolo (1630-1706), 699-841. Thomas, B, GamBer, O y SchedelmanN, H. (1974): Armi e armature Europee. (Edizione italiana a cura di Lionello Giorgio Boccia). Milán.

5 Godoy, J. A. (1987): «Dos armaduras de Eugui para el rey Felipe III (1598-1621)». Reales Sitios 94, 37-44. Godoy, J. A. (1999): "Armeros Milaneses en Navarra: La producción de Eugui». Gladius XIX, 231-260.

6 LaVin, J. D. Ameller, P. (1989): «El regalo de Jacobo I a Felipe III en la Real Armería». Reales Sitios 102, 37-44.

7 Sobre ambos regalos ver las fichas correspondientes en el catálogo de la exposición: El arte en la corte de los Archiduques Alberto de Austria e Isabel Clara Eugenia (1598-1633). Un reino imaginado. (1999) Sala de Exposiciones Temporales del Palacio Real. Madrid. 2 de diciembre de 1999 / 27 de febrero del 2000. Madrid, 194-201. 